\title{
On Eigenvalues and Boundary Curvature of the Numerical Rang of Composition Operators on Hardy Space
}

\section{Mohammad Taghi Heydari}

Department of Mathematics, College of Sciences, Yasouj University, Yasouj, Iran

Email: heydari@yu.ac.ir

Received 9 November 2014; accepted 30 April 2015; published 6 May 2015

Copyright (C) 2015 by author and Scientific Research Publishing Inc.

This work is licensed under the Creative Commons Attribution International License (CC BY).

http://creativecommons.org/licenses/by/4.0/

c) (i) Open Access

\section{Abstract}

For a bounded linear operator $\boldsymbol{A}$ on a Hilbert space $\mathcal{H}$, let $M(A)$ be the smallest possible constant in the inequality $D_{p}(A) \leq M(A) R_{p}(A)$. Here, $p$ is a point on the smooth portion of the boundary $\partial W(A)$ of the numerical range of $A . R_{p}(A)$ is the radius of curvature of $\partial W(A)$ at this point and $D_{p}(A)$ is the distance from $p$ to the spectrum of $A$. In this paper, we compute the $M(A)$ for composition operators on Hardy space $H^{2}$.

\section{Keywords}

\section{Composition Operator, Numerical Range, Eigenvalues, Curvature}

\section{Introduction}

For a bounded linear operator $A$ on a Hilbert space $\mathcal{H}$, the numerical range $W(A)$ is the image of the unit sphere of $\mathcal{H}$ under the quadratic form $x \rightarrow\langle A x, x\rangle$ associated with the operator. More precisely,

$$
W(A)=\{\langle A x, x\rangle: x \in \mathcal{H},\|x\|=1\} .
$$

Thus the numerical range of an operator, like the spectrum, is a subset of the complex plane whose geometrical properties should say something about the operator.

One of the most fundamental properties of the numerical range is its convexity, stated by the famous Toeplitz-Hausdorff Theorem. Other important property of $W(A)$ is that its closure contains the spectrum of the op- 
erator, $W(A)$ is a connected set with a piecewise analytic boundary $\partial W(A)$ [1].

Hence, for all but finitely many points $p \in \partial W(A)$, the radius of curvature $R_{p}(A)$ of $\partial W(A)$ at $p$ is well defined. By convention, $R_{p}(A)=0$ if $p$ is a corner point of $W(A)$, and $R_{p}(A)=\infty$ if $p$ lies inside a flat portion of $\partial W(A)$.

Let $D_{p}(A)$ denote the distance from $p$ to $\sigma(A)$, we define $M(A)$ the smallest constant such that

$$
D_{p}(A) \leq M(A) R_{p}(A)
$$

for all $p \in \partial W(A)$ with finite non-zero curvature.

By Donoghue's theorem $D_{p}(A)=0$ whenever $R_{p}(A)=0$. Therefore, $M(A)=0$ for all convexoid element $A$. Recall that convexoid element is an element such that its numerical range coincides with the convex hull of its spectrum. For non-convexoid $A$,

$$
M(A)=\sup \frac{D_{p}(A)}{R_{p}(A)}
$$

where the supremum in the right-hand side is taken along all points $p \in \partial W(A)$ with finite non-zero curvature.

The computation of $M(A)$ for arbitrary $n \times n$ matrix $A$ is an interesting open problem. For $n>3$, we do not have an exact value of $M_{n}=\sup \left\{M(A): A \in \mathbb{C}^{n \times n}\right\}$. The question whether there exists a universal constant $M=\sup _{n} M_{n}$, posed by Mathias [2]. Caston, et al. [3] prove the following inequalities:

$$
\frac{n}{2} \sin \left(\frac{\pi}{n}\right) \leq M_{n} \leq \frac{n}{2} .
$$

Mirman a sequence of $n \times n$ Toeplitz nilpotent matrices $A_{n}$ with $M\left(A_{n}\right)$ algrowing asymptotically as $\log n$ is also found [3]. Hence, the answer to Mathias question is negative. However, the lower bound in (3) is still of some interest, at least for small values of $n$. The question of the exact rate of growth of $M_{n}$ (it is $\log n$, or $n$, or something in between) remains open.

\section{Composition Operator on Hardy Space}

Let $\mathbb{U}$ denote the open unit disc in the complex plane, and the Hardy space $H^{2}$ the functions $f(z)=\sum_{n=0}^{\infty} \hat{f}(n) z^{n}$ holomorphic in $\mathbb{U}$ such that $\sum_{n=0}^{\infty}|\hat{f}(n)|^{2}<\infty$, with $\hat{f}(n)$ denoting the n-th Taylor coefficient of $f$. The inner product inducing the norm of $H^{2}$ is given by $\langle f, g\rangle:=\sum_{n=0}^{\infty} \hat{f}(n) \overline{\hat{g}(n)}$. The inner product of two functions $f$ and $g$ in $H^{2}$ may also be computed by integration:

$$
\langle f, g\rangle=\frac{1}{2 \pi i} \int_{\partial \mathrm{U}} f(z) \overline{g(z)} \frac{\mathrm{d} z}{z}
$$

where $\partial \mathbb{U}$ is positively oriented and $f$ and $g$ are defined a.e. on $\partial \mathbb{U}$ via radial limits.

For each holomorphic self map $\varphi$ of $\mathbb{U}$ induces on $H^{2}$ a composition operator $C_{\varphi}$ defined by the equation $C_{\varphi} f=f \circ \varphi\left(f \in H^{2}\right)$. A consequence of a famous theorem of J. E. Littlewood [4] asserts that $C_{\varphi}$ is a bounded operator. (see also [5] [6]).

In fact (see [6])

$$
\sqrt{\frac{1}{1-|\varphi(0)|^{2}}} \leq\left\|C_{\varphi}\right\| \leq \sqrt{\frac{1+|\varphi(0)|}{1-|\varphi(0)|}} .
$$

In the case $\varphi(0) \neq 0$, Joel $\mathrm{H}$. Shapiro has been shown that the second inequality changes to equality if and only if $\varphi$ is an inner function.

A conformal automorphism is a univalent holomorphic mapping of $\mathbb{U}$ onto itself. Each such map is linear fractional, and can be represented as a product $w \cdot \alpha_{p}$, where

$$
\alpha_{p}(z):=\frac{p-z}{1-\bar{p} z},(z \in \mathbb{U}),
$$


for some fixed $p \in \mathbb{U}$ and $w \in \partial \mathbb{U}$ (See [7]).

The map $\alpha_{p}$ interchanges the point $p$ and the origin and it is a self-inverse automorphism of $\mathbb{U}$.

Each conformal automorphism is a bijection map from the sphere $\mathbb{C} \cup\{\infty\}$ to itself with two fixed points (counting multiplicity). An automorphism is called:

- elliptic if it has one fixed point in the disc and one outside the closed disc;

- hyperbolic if it has two distinct fixed point on the boundary $\partial \mathbb{U}$, and

- parabolic if there is one fixed point of multiplicity 2 on the boundary $\partial \mathbb{U}$.

For $r \in \mathbb{U}$, an $r$-dilation is a map of the form $\delta_{r}(z)=r z$. We call $r$ the dilation parameter of $\delta_{r}$ and in the case that $r>0, \delta_{r}$ is called positive dilation. A conformal $r$-dilation is a map that is conformally conjugate to an $r$-dilation, i.e., a map $\varphi=\alpha^{-1} \circ \delta_{r} \circ \alpha$, where $r \in \mathbb{U}$ and $\alpha$ is a conformal automorphism of $\mathbb{U}$.

For $w \in \partial \mathbb{U}$, an $w$-rotation is a map of the form $\rho_{w}(z)=w z$. We call $w$ the rotation parameter of $\rho_{w}$. A straightforward calculation shows that every elliptic automorphism $\varphi$ of $\mathbb{U}$ must have the form

$$
\varphi=\alpha_{p} \circ \rho_{w} \circ \alpha_{p},
$$

for some $p \in \mathbb{U}$ and some $w \in \partial \mathbb{U}$.

\section{Main Results}

In [8], the shapes of the numerical range for composition operators induced on $H^{2}$ by some conformal automorphisms of the unit disc specially parabolic and hyperbolic are investigated.

In [9], V. Matache determined the shapes $W\left(C_{\varphi}\right)$ in the case when the symbol of the composition operator the inducing functions are monomials or inner functions fixing 0 . The numerical ranges of some compact composition operators are also presented.

Also, in [10] the spectrum of composition operators are investigated.

This facts will help in discussing and proving many of the results below.

Remark 3.1 If $\varphi=r,|r|<1$, then $\sigma\left(C_{\varphi}\right)=\{0,1\}$ and $W\left(C_{\varphi}\right)$ is a closed ellipticall disc whose boundary is the ellipse of foci 0 and 1, having major/minor axis of length $2 a=\frac{1}{\sqrt{1-|r|^{2}}}$ and $2 b=\frac{|r|}{\sqrt{1-|r|^{2}}}$. Therefore $M\left(C_{\varphi}\right)=\frac{b}{a}=|r|$.

Remark 3.2 If $\varphi=w z,|w|=1, w \neq 1$, then $\sigma\left(C_{\varphi}\right)=$ the closure of $\left\{w^{k}, k=0,1,2, \cdots\right\}$. If $w$ is the $n$-th root of unity then $W\left(C_{\varphi}\right)$ is the convex hull of all the n-th roots of unity and so $M\left(C_{\varphi}\right)=0$. If $w$ is not a root of unity the $W\left(C_{\varphi}\right)$ is the union of $\mathbb{U}$ and the set $\left\{w^{n}: n \geq 0\right\}$. In this case also $M\left(C_{\varphi}\right)=0$.

Remark 3.3 If $\varphi$ is hyperbolic with fixed point $a,|a|<1$, then

$$
\sigma\left(C_{\varphi}\right)=\left\{\lambda: \varphi^{\prime}(a)^{\frac{1}{2}} \leq|\lambda| \leq \varphi^{\prime}(a)^{\frac{-1}{2}}\right\}
$$

and $W\left(C_{\varphi}\right)$ is a disc center at the origin. Therefore $M\left(C_{\varphi}\right)=1-\frac{1}{w\left(C_{\varphi}\right) \sqrt{\varphi^{\prime}(a)}}$ where $w\left(C_{\varphi}\right)$ is the numerical radius of $C_{\varphi}$.

Remark 3.4 If $\varphi$ is parabolic, then $\sigma\left(C_{\varphi}\right)=\partial \mathbb{U}$ and $W\left(C_{\varphi}\right)$ is a disc center at the origin. Therefore $M\left(C_{\varphi}\right)=1-\frac{1}{w\left(C_{\varphi}\right)}$.

Remark 3.5 If $\varphi$ is elliptic with rotation parameter $w$, and $w$ is not a root of unity, then $\sigma\left(C_{\varphi}\right)=\partial \mathbb{U}$ and $W\left(C_{\varphi}\right)$ is a disc center at the origin. Therefore $M\left(C_{\varphi}\right)=1-\frac{1}{w\left(C_{\varphi}\right)}$.

Therefore we have the following table for $M\left(C_{\varphi}\right)$. 


\begin{tabular}{lc}
\hline$\varphi$ & $M\left(C_{\varphi}\right)$ \\
$\varphi(z)=w z,|w| \leq 1$ & 0 \\
$\varphi(z)=r,|r|<1$ & $1-\frac{1}{w\left(C_{\varphi}\right) \sqrt{\varphi^{\prime}(a)}}$ \\
$\varphi \quad$ is a hyperbolic automorphism & $1-\frac{1}{w\left(C_{\varphi}\right)}$ \\
$\varphi \quad$ is a parabolic automorphism & $1-\frac{1}{w\left(C_{\varphi}\right)}$ \\
$\varphi \quad$ is an elliptic automorphism with rotation parameter is not a root of unity & $?$ \\
$\varphi$ is an elliptic automorphism with rotation parameter is a root of unity
\end{tabular}

\section{Completing the Table}

An elliptic automorphism $\varphi$ of $\mathbb{U}$ that does not fix the origin must have the form $\varphi=\alpha_{p} \circ \rho_{w} \circ \alpha_{p}$, where

$$
\rho_{w}(z)=w z(z \in \mathbb{U}),
$$

for some fixed $p \in \mathbb{U}-\{0\}$ and $w \in \partial \mathbb{U}$. If we wish to show this dependence of $\varphi$ on $p$ and $w$, we will denote the elliptic automorphism $\alpha_{p} \circ \rho_{w} \circ \alpha_{p}$ by $\varphi_{p, w}$.

If $\varphi$ is periodic then, surprisingly, the situation seems even murkier: For period 2 has been shown the closure of $W\left(C_{\varphi}\right)$ is an elliptical disc with foci at \pm 1 (Corollary 4.4. of [8]). It is easy to see that $W\left(C_{\varphi}\right)$ is open, also in [11], the author completely determined $W\left(C_{\varphi}\right)$ for period 2.

Theorem 3.6 If $\varphi$ is an elliptic automorphism with order 2 and $P$ it's only fixed point in open unit disc, then there is $0 \leq r \leq 1$ such that

$$
M\left(C_{\varphi}\right)= \begin{cases}\frac{1+|p|^{2}}{2} & \text { if } 0 \leq|p| \leq r ; \\ \frac{2|p|}{1+|p|^{2}} & \text { if } r<|p| \leq 1 .\end{cases}
$$

Proof. Let the operator $A$ be self-inverse, i.e., $A^{2}=I$ but $A \neq \pm I$, so $\partial W(A)$ is an ellipse with foci at \pm 1 [12]. If $\partial W(A)=a \cos (\theta)+i b \sin (\theta)$ with $a^{2}=b^{2}+1$. Then

$$
\begin{aligned}
M(A) & =\sup _{0 \leq \theta \leq 2 \pi} \frac{D(\theta)}{R(\theta)}=\sup _{0 \leq \theta \leq \frac{\pi}{2}} \frac{D(\theta)}{R(\theta)}=\sup _{0 \leq \theta \leq \frac{\pi}{2}} \frac{a b \sqrt{(a \cos (\theta)-1)^{2}+b^{2} \sin ^{2}(\theta)}}{\left(a^{2} \sin ^{2}(\theta)+b^{2} \cos ^{2}(\theta)\right)^{\frac{3}{2}}} \\
& =\sup _{0 \leq \theta \leq \frac{\pi}{2}} \frac{a b(a-\cos (\theta))}{\left.\sin ^{2}(\theta)+b^{2}\right)^{\frac{3}{2}}}=\max \left\{\frac{\sqrt{a^{2}-1}}{a}, \frac{a}{a+1}\right\}
\end{aligned}
$$

If $\varphi$ is an elliptic automorphism with order 2 and $p$ it's only fixed point in open unit disc, then $\varphi=\alpha_{q}$ where $q=\frac{2 p}{1+|p|^{2}}$. Since $C_{\alpha_{q}(z)}$ is a nontrivial self-inverse operator on Hardy space $H^{2}$ and $\alpha_{q}$ is an inner function, then

$$
a=\frac{1}{2}\left(\left\|C_{\varphi}\right\|+\frac{1}{\left\|C_{\varphi}\right\|}\right)=\frac{1}{\sqrt{1-|q|^{2}}}=\frac{1+|p|^{2}}{1-|p|^{2}}
$$


and so there is $0 \leq r \leq 1$ such that:

$$
M\left(C_{\varphi}\right)= \begin{cases}\frac{1+|p|^{2}}{2} & \text { if } 0 \leq|p| \leq r \\ \frac{2|p|}{1+|p|^{2}} & \text { if } r<|p| \leq 1 .\end{cases}
$$

But for period $k>2$ then all we can say is that the numerical range of $C_{\varphi}$ has $k$-fold symmetry and we strongly suspect that in this case the closure is not a disc. Because the numerical range in this case is an open problem, so the completing of $C_{\varphi}$ is also open problem.

\section{Acknowledgements}

I thank the editor and the referee for their comments. Also, when the author is the responsible of establishing Center for Higher Education in Eghlid he is trying to write this paper, so I appreciate that center because of supporting me in conducting research.

\section{References}

[1] Gustafon, K.E. and Rao, K.M. (1997) The Numerical Range, The Field of Values of Linear Operators and Matrices. Springer, New York.

[2] Mathias, R. (1997). http://www.wm.edu/cas/mineq/topics/970103.html

[3] Caston, L., Savova, M., Spitkovsky, I. and Zobin, N. (2001) On Eigenvalues and Boundary Curvature of the Numerical Range. Linear Algebra and Its Applications, 322, 129-140. http://dx.doi.org/10.1016/S0024-3795(00)00231-7

[4] Littlewood, J.E. (1925) On Inequalities in the Theory of Functions. Proceedings London Mathematical Society, 23, 481-519. http://dx.doi.org/10.1112/plms/s2-23.1.481

[5] Shapiro, J.H. (1993) Composition Operators and Classical Function Theory. Springer-Verlag, New York. http://dx.doi.org/10.1007/978-1-4612-0887-7

[6] Cowen, C.C. and Maccluer, B.D. (1995) Composition Operators on Spaces of Analytic Functions. CRC Press, Boca Raton.

[7] Rudin, W. (1987) Real and Complex Analysis. 3rd Edition, McGraw-Hill, New York.

[8] Bourdon, P.S. and Shapiro, J.H. (2000) The Numerical Range of Automorphic Composition Operators. Journal of Mathematical Analysis and Applications, 251, 839-854. http://dx.doi.org/10.1006/jmaa.2000.7072

[9] Matache, V. (2001) Numerical Ranges of Composition Operators. Linear Algebra and Its Applications, 331, 61-74. http://dx.doi.org/10.1006/jmaa.2000.7072

[10] Nordgren, E. (1968) Composition Operators. Canadian Journal of Mathematics, 20, 442-449.

[11] Abdollahi, A. (2005) The Numerical Range of a Composition Operator with Conformal Automorphism Symbol. Linear Algebra and Its Applications, 408, 177-188.

[12] Rodman, L. and Spitkovsky, I.M. (2008) On Generalized Numerical Ranges of Quadratic Operators. Operator Theory: Advances and Applications, 179, 241-256. 